\title{
La Recreación de la Identidad Étnica en la Protesta Mapuche: Un Punto de Partida Para el Uso de la Teoría de Sistemas Sociales en la Politica de la Identidad
}

\author{
Recreation of Ethnic Identitiy in Mapuche Protest: A Starting Point \\ for the Use of the Social Systems Theory in Identity Politics.
}

Salvador Millaleo

\begin{abstract}
RESUMEN
Una critica desde la teoría de sistemas a las reivindicaciones culturales del pueblo mapuche que se basa en la critica de la politica de la identidad cultural, falla en ver la función social de las identidades culturales colectivas. Un enfoque de teoría de sistemas, como el propuesto por Stäheli y Bora, que no deje de lado la función de la cultura como memoria de la sociedad y de las semánticas como generalizaciones de sentido que sirven programarla, incluyendo la etnicidad, y que además supere la relación de jerarquía entre estructura social y semántica, respalda el poder creativo de las construcciones simbólicas para las operaciones de los sistemas sociales, asi como su codeterminación recíproca. A modo de ilustración se estudian los desplazamientos que están ocurriendo mediante una serie de posicionamientos sociales de la politica de identidad mapuche. El movimiento de protesta mapuche en cinco posicionamientos sociales conecta la interpretación que realiza mediante autodescripciones de si mismo con una serie de problemas de la estructuras sociales derivados de programaciones del sistema politico orientadas por el Neoliberalismo y otras consecuencias de la forma concreta de la diferenciación funcional en el Cono Sur. El ejemplo del artículo determina la posibilidad de reformular el concepto de cultura desde las teorias de sistemas sin perder con ello el objeto de la observación.
\end{abstract}

Palabras Clave: Teoría de Sistemas, Politica de la Identidad, Cultura, Movimiento Mapuche, Semántica, Posicionamiento Social.

\begin{abstract}
A criticism, voiced from the systems theory point of view, of the cultural reivindications of the Mapuche people, based on criticism of cultural identity politics, fails to see the social function of collective cultural identities. A focus in systems theory based on Stäheli and Bora, that does not leave aside the function of culture as society's memory and of the semantics as generalisations of meaning that help to program it including ethnicity -, and the furthermore is able to overcome the hierarquical relationship between social structure and semantics, supports the creative power of symbolic constructions for the operatings of the social systems, as well as their reciprocal codetermination. As an illustration, displacements that take place via a series of social positionings of Mapuche identity politics are studied. The Mapuche protest movement, in five social positionings, connects the interpretation it makes of itself via autodescriptions with a series of problems in the social structure, derived from programations of the political system oriented in Neoliberalism, as well as other consequences of the concrete form of functional differentiation in Southern Cone. The example analysed in the article looks into the possibiliy of reformulating the concept of culture within social system theory without losing its objetc of observation.
\end{abstract}

Keywords: Systems Theory, Identity Politics, Culture, Mapuche Movement, Semantics, Social Positioning.

Universidad Diego Portales. República 105, Santiago. Correo-e: smillaleo@hotmail.com

Recibido: mayo 2010. Aceptado: diciembre 2010. 


\section{INTRODUCCIÓN}

En la actualidad no constituye una sorpresa la denegación de los reclamos de la política de la identidad. Esto ha llegado a ser un tópico para confrontar la explosividad y globalización de estas políticas. Aquella estrategia responde a la sospecha sobre la política de la identidad en cuanto ésta representa un peligro para la mantención de diferenciaciones constituidas hegemónicamente, pues las cuestiona abiertamente. Como indica Alcoff (2000: 312-313), "muchos teóricos están problematizados por las implicaciones de que la identidad hace una diferencia. Crecientemente la adhesión a una identidad ha llegado a ser sospechosa. Si la identidad ha llegado a ser sospechosa, la política de la identidad ha sido perseguida, juzgada y sentenciada a muerte".

Mascareño (2007a), en su análisis de la encuesta aplicada por el Centro de Estudios Públicos (CEP) en 2006 y cuyos resultados mostraron que las preferencias de los mapuches y no-mapuches no difieren substancialmente entre sí, criticó la reclamación de una cultura mapuche en Chile desde la teoría de sistemas. Las diferencias reales aparecen en el terreno político. Por ello, el autor concluye que las demandas de una cultura mapuche son descriptivamente pobres, pero políticamente ricas, quizás demasiado ricas. Sostiene que "la cultura no puede ser invocada como categoría sociológica para dar cuenta de la condición actual de este grupo étnico, aunque cuando se trata de impulsar, construir o exhortar a tal diferencia étnica, el concepto puede adquirir alta relevancia a modo de autodescripción política" (Mascareño 2007a: 62).

Más allá del caso específico de los mapuches, Mascareño (2007a), argumenta que en una sociedad funcionalmente diferenciada e individualizada, como parece ser la chilena, la aglomeración de diferencias que resume el concepto de cultura es sólo una ilusión, aunque una ilusión políticamente productiva. Por lo tanto, él intenta una deconstrucción de los reclamos culturales de los activistas mapuches para hacer una ilustración sociológica de aquella ilusión. Esta ilustración incluye la crítica del concepto de cultura, el cual sólo es útil para la descripción de sociedades segmentarias y estratificadas, pero ya no para la moderna sociedad mundial, la cual está funcionalmente diferenciada. Las estructuras sociales de la sociedad mundial disuelven las identidades integradas e inducen a la dispersión de las posibilidades de inclusión individualizadas (interpenetración) y a la contradicción de las resultantes identidades fragmentadas (inconsistencias) (Mascareño 2007a). En consecuencia, desde el punto de vista de la teoría de sistemas, y según 
este autor, hablar aún de cultura e identidad en las ciencias sociales es lo mismo que hablar del éter en el vacío. A pesar de esos problemas analíticos, la ficción de la identidad serviría a los sujetos para describirse a sí mismos como culturalmente diferenciados para incrementar y hacer más probables sus posibilidades de inclusión funcional en los sistemas sociales.

En un ensayo posterior, Mascareño (2007b) explica la cultura como una ficción real, ficticia en cuanto construcción simbólica en el medio del sentido que presenta una unidad de la observación de primer orden que funciona como una autodescripción del observador. Para él, toda descripción cultural tiene por pretensión una unidad que supone, como condición de verosimilitud, un riesgo para ella, una heterogeneidad, una diferencia con la unidad que la descripción cultural describe. Sería, sin embargo, real en cuanto a las consecuencias concretas de las posibilidades de inclusión y exclusión que ofrece. La descripción cultural sería un dispositivo de comunicación para posibilitar el éxito del acoplamiento entre individuos y sistemas, es decir, la inclusión. En el caso de los mapuches, la fórmula "cultura mapuche" podría diseñarse para mejorar la inclusión de los miembros englobados en ella sobre la base de definir a la vez como ineludibles y amenazados ciertos contenidos de la descripción. La "permanencia" de la cultura mapuche en su descripción está destinada a hacer más plausible la inclusión de los individuos en base a los méritos de los elementos de la descripción (Mascareño 2007b).

A nuestro entender, este enfoque de teoría de sistemas de la política de la identidad queda limitado frente a las herramientas que esa misma teoría ofrece para comprender las identificaciones sociales de los individuos y las construcciones identitarias colectivas. Simultáneamente, reduce el alcance de la política de la identidad en orden a obtener posibilidades de inclusión individualizadas, lo cual no presta atención a la función de las estructuras comunicativas de las identidades colectivas en los acoplamientos estructurales entre el sistema político y los otros sistemas sociales, por ejemplo, los movimientos sociales, la religión, los grupos étnicos y los medios de comunicación. El enfoque mencionado falla en apreciar la función de la etnicidad, el género y otras semánticas de la identidad en los modernos sistemas sociales y pierde la perspectiva respecto de importantes estructuras de intermediación para la auto-producción de la comunicación en dichos sistemas, tales como las formaciones discursivas y los posicionamientos sociales. La movilidad y el poder de las identidades colectivas pertenecen a las características principales de la moderna y funcionalmente diferenciada sociedad mundial. Este ensayo intenta una aplicación de una perspectiva más amplia de la teoría de 
sistemas. Posteriormente, se buscará ilustrar este enfoque mediante su aplicación preliminar a la política de la identidad del movimiento de protesta mapuche en Chile y Argentina.

\section{IDENTIDADES CULTURALES Y AUTO-POSICIONAMIENTOS EN UNA} SOCIEDAD MUNDIAL FUNCIONALMENTE DIFERENCIADA

El antecedente de la citada deconstrucción de lo mapuche es la actitud temprana de Niklas Luhmann hacia el concepto de cultura, quien tuvo una actitud muy negativa respecto a la tradición de este término. Es bien conocida su afirmación que el concepto de cultura es uno de los peores conceptos que las ciencias sociales jamás hayan formulado (Luhmann 1995: 398). A pesar de tal afirmación, él desarrollará otros enfoques para conceptualizar la cultura, ocurriendo una transformación del significado teórico del término (Zimmerman 2003: 44).

En primer lugar, conceptualiza la cultura como memoria de la sociedad (Luhmann 1995, Espósito 2002). Como memoria de la sociedad toda, la cultura no construye un archivo o reserva de identidad, sino que pone a prueba los programas de los sistemas sociales con las informaciones de sus entornos. Sería la variación estructural, antes que la defensa de una identidad, lo que caracterizaría la memoria cultural de la sociedad (Baecker 2003). La variación estructural ha sido explicada con el postulado de la subordinación coevolutiva de la semántica a las estructuras sociales (Luhmann 1980, 2008). Relacionado a ello, Luhmann (1984) identificó la cultura como el conjunto de los órdenes semánticos de la sociedad.

En segundo lugar, la cultura podría ser conceptualizada como una observación de segundo orden de índole comparativa y diferenciadora (Luhmann 1997, 1999b; Baecker 2003). La cultura es una forma de observación y autodescripción de la sociedad (Baecker 2003), cuyas operaciones describen diferencias comparativas. La cultura ya no consistiría en patrones o valores sociales, sino que en diferencias y la operación de comparación sobre la base de esas diferencias. La contingencia moderna posibilita la comparación, de manera que el contenido y forma de las comparaciones culturales permanecen siempre contingentes. La cultura como descripción comparativa no partiría entonces de la unidad y permanencia, sino de la diferencia y la contingencia (Schroer 2010). Si bien las descripciones culturales son básicamente autodescripciones, como operaciones comparativas no constituyen una observación de primer orden, sino de segundo orden que siempre será interpretada por otro observador, de manera que de toda perspectiva cultu- 
ral resultaría una ambivalencia, debido a la posibilidad de otra observación (Schroer 2010), así como de toda cultura resultaría un contacto, un contacto con la diferencia con la cual se compara (Baecker 2008).

La memoria sistémica facilita la continuidad de los sistemas mediante sus cambios al conectar el presente con el pasado de la historia del sistema. Ella es distinta que la agregación de las memorias individuales de los participantes en la comunicación social, es una propiedad del sistema. La función de esta memoria es vincular las nuevas operaciones del sistema con las antiguas. Las nuevas operaciones son posibles como selecciones sobre la base de la aplicación del filtro recordar/olvidar a las viejas selecciones (Espósito 2002).

La memoria al nivel del sistema de la sociedad en su conjunto, como a nivel de los otros sistemas sociales (interacciones, organizaciones, grupos), se organiza a sí misma en dos elementos: Estructuras sociales y semánticas. Aunque esta distinción no es formulada claramente en la obra de Luhmann (Stichweh 2000), esta distinción puede ser relacionada con la diferencia entre operaciones simples y operaciones de observación (Stäheli 2000). Semánticas y estructuras son construcciones generalizadas de sentido. Sin embargo, la semántica representa generalizaciones de construcciones simbólicolingüísticas, mientras que las estructuras sociales son sentido silenciosa y anónimamente organizado.

Luhmann construyó un modelo en el cual la semántica coevoluciona con las estructuras sociales, pero de una manera en que la semántica elabora simbólicamente y expresa las transformaciones en la estructura social. Por su parte, la estructura social evoluciona de acuerdo con los cambios en la forma de diferenciación de la sociedad.

Stäheli (2000) ha criticado este modelo en tanto que jerárquico y ha propuesto uno nuevo. Las semánticas serían construcciones de las observaciones de la sociedad, las cuales no sólo tienen un rol expresivo o reconstructivo, sino también uno constitutivo. Las autodescripciones que proporciona la semántica son fundamentales para la realización de las operaciones del sistema, tales como la definición de la identidad de los actores sociales, la determinación de los tópicos de comunicación y las interpretaciones temporales y locales. Sin embargo, la semántica no puede ser considerada como un proceso integrado y unificado que ofrecería una unidad indiscutible y una identidad única a los sistemas sociales. La auto-producción de la comunicación en los sistemas sociales es un proceso plural y agonístico de determinación de los programas del sistema. No existe una sola tradición se- 
mántica, sino muchas semánticas que ofrecen versiones para la construcción de las operaciones del sistema. Esta pluralidad de alternativas semánticas se relaciona con una lógica evolutiva propia de las representaciones simbólicas que no depende solamente de las transformaciones de las estructuras sociales. Los imaginarios sociales definen las comunicaciones en su concurrencia cuando, por una parte, son soportadas por estructuras sociales favorables $\mathrm{y}$, por otro lado, alcanzan una posición simbólica hegemónica frente a otras alternativas. En cualquier caso las semánticas o discursos junto a las estructuras sociales derivadas de la diferenciación social son recíprocamente constitutivos de lo social (Stäheli 2000).

La concepción sistémica de cultura recién referida puede perfectamente dialogar con nociones que han superado las concepciones clásicas y descriptivas de cultura (Thompson 2002). En particular, la superación del modelo jerárquico de la relación entre semánticas y estructuras sociales hace posible para la teoría de sistemas un diálogo productivo con los Estudios Culturales y sus derivados (como los Estudios Postcoloniales). Otro paso en dicha dirección consiste en la reflexión sobre la formación de esquemas comparativos de la cultura. La forma comparativa de las construcciones culturales está conectada al posicionamiento social relacional de los sujetos en las comunicaciones sistémicas.

Aunque la teoría de sistemas argumenta que el concepto fundamental para la descripción de la sociedad es la comunicación y que los sujetos son relevantes sociológicamente sólo como participantes en comunicaciones, mientras el procesamiento subjetivo de sentido permanece en el dominio psíquico, esta teoría asume que existe una coevolución constitutiva entre sistemas sociales y sistemas psíquicos. La construcción de los destinatarios en la comunicación, la atribución de acciones y responsabilidades, y de identidades sociales a individuos, así como otros procesos subjetivos, son construcciones sociales. Ellos son posibles sólo a través de múltiples semánticas y estructuras de comunicación. Esto se aplica no solamente a identidades individuales sino que también a identificaciones colectivas, sea que éstas estén estrechamente relacionadas con la identidad del sistema (como en las identidades organizacionales o de grupo), o que éstas están construidas dentro de comunicaciones flotantes, es decir que no están limitadas a un sistema (como identidades étnicas difusas, de género o morales).

Las construcciones sociales de identidades están relacionadas con auto-posicionamientos relacionales en conversaciones y otras formas de comunicación (Davies y Harre 1990). Según estos autores, un individuo emerge 
mediante los procesos de interacción social, no como un producto final fijo, sino como constituido y reconstituido a través de las variadas prácticas discursivas en que ellos participan. Correspondientemente, el sujeto es siempre una pregunta abierta con una respuesta variable dependiendo de las posiciones disponibles dentro de prácticas discursivas propias y ajenas, $\mathrm{y}$, dentro de aquellas prácticas, de las narraciones mediante las cuales damos sentido a nuestras vidas y a las vidas de otros. Las narraciones están situadas dentro de múltiples y diferentes discursos.

Para la construcción del yo e interpretación del mundo desde esa perspectiva, es central el aprendizaje de las categorías que incluyen a unos y no a otros como masculino/femenino, etc. La participación en la comunicación de sujetos realiza y supone la adjudicación de sentido a aquellas categorías. El Yo es entonces posicionado en relación a tramas de sentido (storylines) que son articuladas en discursos en torno a esas categorías. Posicionamiento significa consecuentemente,

\begin{abstract}
el proceso discursivo por el cual las identidades del yo son emplazadas en conversaciones como participantes observables y subjetivamente coherentes en tramas de sentido producidas simultáneamente. Puede haber un posicionamiento interactivo en el cual lo que una persona dice posiciona a la otra. Y puede haber un posicionamiento reflexivo en el cual uno se posiciona a si mismo" (Davies $y$ Harre 1990: 48).
\end{abstract}

Identidades portátiles (ocasionales) y fundamentales (permanentes) tienen procesos de construcción generales mediante el posicionamiento del yo y de los otros, del mismo modo que las identidades colectivas, en las cuales los sujetos individuales reflexionan simultáneamente sobre sus posiciones respecto a los demás y respecto a su pertenencia a un grupo o categoría social. En cuanto los colectivos organizan sus propios puntos de comunicación, ellos pueden comunicar y posicionarse a sí mismos y a otros (otros colectivos o individuos en relación a colectivos) en sus prácticas discursivas (Moghaddam 2007). Por lo anterior, el rol del discurso en la estructuración de las comunicaciones puede ser situado en la organización de la memoria de la sociedad y los demás sistemas sociales.

Los nuevos enfoques de la teoría de sistemas sociales argumentan que es útil la inclusión de la categoría discurso en su reflexión teórica (Bora 1999, Stäheli 2000). El discurso es una estructura selectiva que puede organizar 
todos los aspectos de las comunicaciones, tales como secuencias temporales, establecimiento de temas e identidades sociales de los participantes en la comunicación (individuales y colectivas) (Bora 1999). Los discursos se diferencian dentro de los sistemas sociales y dependen de la lógica de reproducción propia de esos sistemas. Ellos son, en cualquier caso, plurales y múltiples, y mantienen relaciones de exclusión con otros discursos, de manera que los procesos de auto-producción de los sistemas sociales a través de las autodescripciones son conflictivos y marcados por la rivalidad. Los conceptos de discurso y posicionamiento social pueden, por lo tanto, asumir la tarea de describir las relaciones entre cultura y sociedad, y entre sociedad y sujeto, dentro de la teoría de sistemas (Bora 2008).

Con relación a las identidades colectivas, ambos conceptos no contradicen la tesis de una sociedad mundial funcionalmente diferenciada, sino que la diferenciación de formaciones discursivas y posicionamientos sociales múltiples y flexibles es solamente posible como un producto de la diferenciación funcional. Dicha sociedad mundial es una sociedad en la cual los sistemas funcionalmente especializados como la economía y la ciencia escapan de las fronteras nacionales, lo cual no excluye las identificaciones culturales como naciones o pueblos. Por el contrario, la sociedad mundial usa esas identificaciones culturales, por ejemplo, en las semánticas étnicas, para la formación de sus propias estructuras (por ej. la soberanía de los Estados se ha basado fundamentalmente en identidades étnicas, así como también los derechos de minorías).

Adicionalmente, no es difícil apreciar cómo la individuación de las identidades sociales en una sociedad funcionalmente diferenciada no excluye la funcionalidad de la semántica de las identidades colectivas como la etnicidad. La etnicidad hace posible la inclusión del individuo como persona en su conjunto y no sólo como puntos temporales de comunicaciones funcionales (como consumidores para la economía, estudiantes para la educación, votantes para la política, sujetos jurídicos para el derecho o investigadores para la ciencia). Precisamente porque las semánticas de integración tradicionales como la religión y el estatus no resultan más adecuadas para una sociedad funcionalmente diferenciada, pueden surgir nuevas y más flexibles formas de semánticas inclusivas (Nassehi 1990). La modernidad del nacionalismo es por ello indiscutible. Esto incluye la forma "integrativa" del nacionalismo cívico, tanto como la forma "divisiva" del etno-nacionalismo, porque ambas son construcciones discursivas hegemónicas que se basan en narrativas y posicionamientos colectivos. 
Los discursos de la etnicidad y los posicionamientos étnicos se relacionan usualmente con estructuras de clase, género y estructuras políticas, y construye de esa forma fenómenos sociales etnificados muy complejos. Las semánticas étnicas son inclusivas y a la vez flexibles y porosas. La narratividad de las construcciones étnicas cambia y se mueve dentro de sus diferencias internas (Bhabha 1990). El nacionalismo y otras representaciones étnicas son construcciones híbridas y ambivalentes. Ambivalentes porque sus narraciones, ritos y rituales están cambiando constantemente; híbridas porque los discursos que trazan las periferias imaginadas en torno a las etnicidades son constantemente hechas y rehechas. Siempre existe la posibilidad de cruzar las fronteras que dividen los de aquí y los de allá. Siempre existiría la posibilidad de incluir y excluir nuevas voces y crear nuevos sitios de sentido, y de generar nuevas relaciones sociales. Bhabha (1990) sostiene que el holismo de las comunidades étnicas es perturbado por la diferencia cultural, la cual rearticula en diferentes maneras los sentidos de los signos del discurso dominante dentro de aquellas comunidades. La diseminación de las configuraciones étnicas a través de la contingencia y complejidad está también caracterizada por los actuales flujos culturales de la sociedad mundial que son posibilitados por las industrias culturales, las migraciones y otras movilidades -corporal, física, imaginativa, comunicativa y virtual (Urry 2002)-, la construcción de espacios transnacionales, la difusión de los medios globales, etc. Las identidades en sociedades funcionalmente diferenciadas son, en términos de la teoría de sistemas sociales, altamente contingentes, en tanto la alteridad, fluidez, multiplicidad y condición de ser construidas no pueden ser reducidas. De acuerdo a Nassehi (2006), la paradoja de la cultura como un esquema de observación reside en el énfasis en la contingencia, porque las formaciones culturales, como las identidades colectivas, no pueden controlar y determinar totalmente la exclusión de observaciones alternativas.

Las semánticas étnicas, aun en sociedades que presentan un primado de la diferenciación funcional, ofrecen posibilidades de posicionamiento social a los sujetos que participan en sus discursos. Dichos posicionamientos individuales y colectivos pueden tener efectos de inclusión o exclusión respecto de sistemas funcionales, o de otro tipo, por ejemplo, en la programación de los sistemas funcionales. Tal es el caso del sistema político mediante las políticas de identidad. 


\section{IDENTIDADES Y ESENCIALISMO EN LA POLÍTICA DE LA IDENTIDAD}

Benhabib (2002: 1) ha indicado que "la cultura ha llegado a ser un sinónimo ubicuo para la identidad, un marcador de identidad y un diferenciador". La cultura y las identidades culturales funcionan como marco de referencia para las comunicaciones políticas. De allí es precisamente que nace el concepto de política de la identidad, el cual si bien puede ser identificado como un conjunto de estructuras y discursos que operan en varios sistemas, ha recibido su primera orientación hacia el sistema político, al cual busca programar desde la periferia hacia el centro de éste.

La política de la identidad lleva la identidad a la esfera pública desafiando el supuesto liberal de la separación de lo particular respecto de lo universal. De acuerdo a Heckman (2004), esto descorre el velo de la ciudadanía abstracta y neutral del liberalismo y revela su identidad contextualizada, dirigiéndose hacia una ciudadanía más bien arraigada en su contexto. La política de la identidad nace de la crítica de muchos nuevos movimientos sociales a los supuestos universalistas de una política común, los cuales son ciegos sobre las consecuencias opresivas de la raza, el género, la edad, la habilidad, la nacionalidad y la religión, entre otros (Young 2000). Los nuevos movimientos sociales étnicos reclaman de los programas de la política una mayor consideración al efecto de las semánticas de la etnicidad en los procesos políticos.

Los discursos de la política de la identidad enfatizan a menudo el valor de la diversidad para la democracia (Charles Taylor, Will Kymlicka, Iris Young, Axel Honneth, Nancy Fraser). La inclusión de la diversidad mediante políticas de reconocimiento y redistribución fomenta la recepción de todos los intereses legítimos y los valores en la política y permite la mantención y desarrollo de marcos de significado para la participación política de los ciudadanos. Esto favorece una igualdad que es sensible a toda clase de diferencias y que no es ciega respecto a los sistemas tradicionales de opresión que la democracia liberal no elimina. La política de la identidad se focaliza en las controversias en marcos significativos y sobrepasa las descripciones de la política como composición de intereses. Además, la reelaboración de la memoria social sobre relaciones pasadas de índole asimétrica y desigual mediante la política de la identidad puede hacer más complejos los acuerdos de justicia de long dureé en los sistemas políticos. Finalmente, la política de la identidad provee a la política y otros sistemas sociales de alternativas más diversas que la política ideológica o de poder de las democracias liberales. 
Por lo anterior, la política de la identidad puede ser un recurso fundamental para la democracia.

Una primera definición de las políticas de la identidad ha conectado los posicionamientos étnicos con reclamos normativos, reclamando políticas especiales, dirigidas a reconocer derechos individuales y colectivos, y a establecer políticas públicas que tengan por objetivo mejorar las condiciones sociales de inclusión de grupos de personas en diversos sistemas funcionales. A menudo dichas pretensiones han sido acompañadas de descripciones culturales que suponen que las culturas son conjuntos claramente delineables, coextensivos a la existencia de determinados grupos, en los cuales serían posibles descripciones identitarias no controvertidas, y que no es representable el problema de un grupo con diversas culturas y diversos grupos que compartan una cultura (Benhabib 2002). Bajo esta lógica, la política de la identidad sería propiedad de un grupo determinable que es definido por un conjunto de atributos esenciales. El grupo compartiría necesariamente esos atributos (Young 2000). Estas descripciones pueden ser observadas con el concepto de esencialismo identitario y no son la única posibilidad de las políticas de identidad.

El esencialismo identitario congela la fluidez y la multiplicidad de la identidad estableciendo distinciones rígidas hacia afuera y adentro (Young 1997). Sin embargo, las identidades nunca han sido representaciones de un yo real, y aquella interpretación siempre fallará, aun cuando tenga éxito en disciplinar las propias prácticas (Alcoff 2000). Las identidades son construcciones discursivas y cambian con la diseminación de sus discursos (Butler 1990). Adicionalmente, aunque los sujetos que comparten su pertenencia a grupos sociales oprimidos y discriminados, convergen en las afinidades políticas, descripciones de otros y autodescripciones, ellos expresan usualmente en un nivel más concreto intereses y valores divergentes e incluso contradictorios (Young 2000).

En América Latina, la formación de nuevos movimientos sociales indígenas y afrodescendientes durante el siglo XX ha reflejado la contingencia de las identidades étnicas mediante su desplazamiento, descentramiento y evidencia de su construcción relacional por medio de políticas de identidad que a menudo tematizan de manera entrelazada semánticas étnicas con semánticas de la religión, género y otras expresiones culturales (Wade 1997). El despliegue de dichas identidades mediante políticas de movimientos étnicos de protesta asumió la posición fundamental de resistencia frente al fracaso de semánticas de integración que han usado los programas de los 
sistemas políticos de la región, desde los programas nacional-populares a los neoliberales (Escobar 1992). Yashar (2005) llama a esos programas regímenes de ciudadanía, atendiendo a sus efectos de inclusión y exclusión social de base étnica, explicando que su transformación ha resultado en la politización de las diferenciaciones étnicas a partir de la defensa y construcción de autonomías locales, permeando los procesos políticos nacionales e internacionales. Los movimientos étnicos han asociado entonces su protesta en la región tanto al cuestionamiento de los legados coloniales y sus derivaciones relativas a la violencia, opresión y exclusión contra los pueblos indígenas y afrodescendientes (Quijano 2000), como a la construcción y defensa de arreglos institucionales de autonomía dentro del Estado (Díaz-Polanco 1997).

\section{El Caso del movimiento mapuche en Chile y Argentina: POSICIONAMIENTOS.}

El movimiento de protesta del pueblo mapuche en el Cono Sur es precisamente un buen ejemplo de la construcción de semánticas contingentes de lo étnico. Dichas semánticas permiten posicionamientos políticos mediante políticas de identidad, las cuales tematizan el legado postcolonial de exclusión en la operación de los sistemas funcionales y pretenden derechos colectivos autonómicos para contrarrestar las lógicas excluyentes de los sistemas políticos y económicos (cabe agregar aquí también el sistema de medios y el orden jurídico). Dichas semánticas no sólo tienen un efecto real en cuanto a los derechos de autonomía perseguidos, sino que corresponden a discursos y posicionamientos sociales de lo mapuche que son operados en aquellas políticas de la identidad. El concepto fundamental para ilustrar un análisis sistémico del movimiento mapuche -un mayor desarrollo en esa vía no es el objeto de este ensayo- será el de posicionamiento social. El posicionamiento siempre implica la constitución de un sujeto que mediante sus efectos discursivos se posiciona frente a diferencias que se relacionan con otras diferencias de acuerdo a las selecciones que realiza el sistema en su observación.

El movimiento mapuche corresponde a un sistema de protesta de la sociedad, autonomizado de otras formas de comunicación (política, artística o religiosa) sobre lo mapuche, y como tal ha posicionado autodescripciones y descripciones de otros de base étnica, las cuales constituyen esquemas comparativos contingentes propios de operaciones de una observación de segundo orden. Los discursos de las organizaciones mapuches discuten críticamente, por ejemplo, la falta de ciudadanía, la pobreza y la margi- 
nalidad de los mapuches en relación a las poblaciones argentina y chilena como resultado del modelo de construcción del Estado realizado en el Cono Sur, la invasión del modelo capitalista y la exclusión mediática; es decir, las consecuencias del concreto modo en que se han producido las operaciones de los sistemas funcionales de la política, la economía y los medios. Esto establece también una continuidad de memoria histórica entre los mapuches del presente y sus raíces históricas precoloniales, pero sobre todo estas tematizaciones en la comunicación de la protesta mapuche se interrelacionan con las elaboraciones identitarias que producen los mapuches dentro de esas mismas comunicaciones.

Las diseminaciones de las diferencias étnicas son procesadas discursivamente, en orden a la producción de una renovación de la identidad mapuche en relación a las identidades nacionales y en el contexto de un mundo globalizado. Dichas diseminaciones reelaboran los bordes externos y los quiebres internos de la identidad mapuche, la cual puede ser entonces manipulada, precisamente porque se ha vuelto contingente en una sociedad mundial funcionalmente diferenciada.

Podemos referir prima facie cinco posicionamientos generales que han sido formulados por la política de identidad de la protesta mapuche, sin que esto agote o excluya otros posicionamientos a niveles macro, micro y meso que presentan sus propias selecciones, variaciones y estabilizaciones.

\section{Posicionamientos sobre la memoria.}

En primer lugar, el surgimiento de los movimientos mapuches a ambos lados de los Andes interroga la memoria del sistema de la sociedad, cuestionando las estructuras discursivas que han legitimado la incorporación de los indígenas al proyecto de los Estados nacionales bajo nombres como "Pacificación de la Araucanía" (León 2007) y "Conquista del Desierto". De acuerdo a Toledo (2006a: 14), "La memoria histórica ha sido un recurso fundamental para el proceso de constitución del movimiento mapuche como actor político, e interpelar a la sociedad y Estado chileno y exigir el reconocimiento y respeto de sus derechos". La intervención de las organizaciones indígenas desde la transición chilena -aunque también esto es visible antes (Bengoa 1991, Foerster y Montecino 1988)- ha estado claramente orientada por políticas de la memoria donde el posicionamiento político se relacionaba con un posicionamiento respecto a la memoria oficial que había expulsado la diferencia mapuche de las semánticas de la unidad e identidad nacionales (Foerster 2001). 
En Argentina, por su parte, la discusión sobre la memoria oficial ha sido una de las cuestiones básicas de la legitimación de las organizaciones mapuches como actores, dado el cuestionamiento de los mapuches como invasores extranjeros en las pampas argentinas para excluir su condición de pueblo originario y reivindicar la legitimidad pura de la anexión (Trentini et al. 2010). De esa manera, la política de identidad mapuche interviene adversarialmente la memoria de la sociedad y propone su fluctuación, de una manera que les permite legitimarse como portadores y guardianes de esa memoria histórica excluida de las semánticas nacionales, a la vez que promotores de una nueva memoria social que posibilite selecciones que hasta el momento el sistema político no es capaz de hacer, como el genuino reconocimiento de los pueblos indígenas.

Desde los setenta, surgieron organizaciones que querían movilizar a los mapuches del Puelmapu (Sector oriental de los Andes del Sur: Argentina) y crecieron dentro de los movimientos de democratización argentina en los ochenta. En el quinto centenario del viaje de Colón muchas organizaciones convergieron en la propuesta "Tai-Ki-e Getuam" (Volver para ser uno), la que formuló demandas de reconocimiento para los mapuches en Argentina que incluían la autodeterminación y los derechos de autonomía (Briones 2006). El Estado argentino adoptó el Convenio $N^{\circ} 169$ (OIT/1989) en 1992, mientras que Chile sólo introdujo en 2008 esa convención internacional en su sistema legal.

En efecto, las élites políticas nacionales en Chile fueron largo tiempo reluctantes a la implementación de una política de reconocimiento para los mapuches (Van Bebber 2002). Salazar (1999), explica esa reluctancia por el miedo a que dicho reconocimiento puede socavar la clásica visión de una nación en un Estado. A pesar de acuerdos iniciales entre los gobiernos democráticos y las organizaciones mapuches, una política de reconocimiento integral no pudo tomar forma y la política indígena fue reducida a satisfacciones limitadas a demandas de recuperación de tierras de comunidades rurales, lo cual dará oportunidades y temas a las comunicaciones de protesta mapuche.

\section{Posicionamientos sobre la condición de actor.}

En términos de la teoría de sistemas (Luhmann 1997, Hellman 1996), las organizaciones mapuches en Gulumapu y Puelmapu construyeron un movimiento de protesta contra las sociedades argentina y chilena, el cual ha autonomizado su comunicación respecto a los actores del sistema político. Las organizaciones mapuches han construido sus propias estructuras 
y permanecen independientes de los actores políticos de Argentina y Chile. Los programas de dichas organizaciones no se apoyan en la confianza en los partidos y las coaliciones, en cuanto estos han quebrado sus promesas y han mantenido sus perspectivas asimilacionistas. De esa manera, la condición de actor no proviene de los discursos del sistema político, donde el indigenismo no ha sido parte de las semánticas del sistema, sino de comunicaciones que ocurren en su periferia y que poseen su propia condición de emergencia en la lógica de la protesta mapuche. El movimiento mapuche reclama además, como otros movimientos indígenas, una autonomía epistemológica, en el sentido que reivindicarían formas propias de conocer e interpretar del mundo y entorno en el cual habitan y se reproducen, distintas a las de los actores del sistema político (Valdés 2007).

La función de la protesta mapuche no es otra que la crítica de la incompletitud de los procesos de diferenciación funcional en el Cono Sur, en cuanto, como sistemas políticos poscoloniales, permanecen sobrecargados con las exclusiones políticas masivas de base étnica y son reducidos por políticas neoliberales a la impotencia para regular la lógica predatoria de la economía que usa ahora esas exclusiones en beneficio de su propia determinación sistémica.

\section{Posicionamientos sobre la identidad: Esencialismo, Reterritorialización, Diáspora y Globalización.}

El movimiento mapuche ha tenido por objetivo el fortalecimiento de la identidad mapuche (Marimán 2003), la que se posiciona frente a las identidades chilena o argentina. Las nociones del retorno de la identidad mapuche y sus procesos de construcción etno-nacionales (Foerster 1999) han sido interpretadas por algunos de los actores relevantes del movimiento mapuche desde una matriz esencialista, que asume a veces contenidos cósmicos y otras nostálgicos. Dicha perspectiva se nutre de los posicionamientos reactivos y defensivos frente a la denegación social de la diferencia mapuche (Para Chile ver Kotov y Vergara 1995, para Argentina Kropff 2005). De acuerdo a Bretho e Iriarte (2003), esta defensa se caracteriza por sustentarse en principios, valores y atributos que son interpretados como fundamentales para la existencia particular y, por lo tanto, son en propiedad intransables. Sin embargo, otras perspectivas, como la diaspórica-urbana, la neoterritorial o la global-mediatizada están desde hace tiempo jugando un rol importante en la construcción de los discursos de identidad mapuches. 
La reterritorialización o neoterritorialismo de los discursos identitarios mapuches ha sido característico de la evolución de los posicionamientos mapuches en los últimos tiempos (Le Bonniec 2002). Este desplazamiento reivindica espacios territoriales ancestrales dentro del territorio nacional. Esto ha sido más evidente en el lado chileno en cuanto a las identidades como la de los Pewenches, Nagche o Lafkenches. Tales identidades son consideradas espacios de reconstrucción de identidad mapuche a la vez que de posicionamiento dentro de ella. El movimiento mapuche deconstruye así las fronteras administrativas y estatales y presenta nuevas cartografías con las cuales reconstruyen (o reinventan) diferenciaciones locales. En Argentina, la delimitación y redenominación del Puelmapu como territorio mapuche compite con los límites provinciales designados por los Estados en los imaginarios del movimiento mapuche.

Mientras tanto, en ambos lados de los Andes la diáspora de individuos mapuches en centros urbanos lejanos a los territorios ancestrales ha llegado a ser un tema crucial. Lo que en los primeros tiempos de la construcción del Estado fue mostrado como una irreversible pérdida de la identidad mapuche y la integración en nuevas pautas de identificación nacional (Argentina y Chile), ha llegado a producir un nuevo espacio étnico (Aravena 1999, Bello 2002, Peyser 2003, Antileo 2006, Millán 2008). La emergencia de los Mapurbe o Warriache, esto es, los habitantes mapuches de las grandes ciudades con organizaciones identitarias e instituciones y prácticas etnoculturales, transforman gradualmente las autodescripciones de la identificación mapuche. En el espacio urbano surgen denominaciones como mapunkies, mapuheavies y mapurbes que expresan la acción de espacios étnicos de fusión o fricción, como prefiere decir Briones (2007).

Por otra parte, se ha evidenciado una globalización de los marcos interpretativos del discurso en el hecho de que muchos conceptos, estrategias y tropos del movimiento mapuche provienen del movimiento indígena global, el cual ha estado comprometido con ideas de autodeterminación, autonomía, interculturalidad y derechos multiculturales, etnodesarrollo, empoderamiento, descentralización, participación deliberativa, etc. El lenguaje del movimiento mapuche para la recreación de la identidad mapuche es un lenguaje global que viaja con las conexiones de un espacio público global incipiente en la sociedad mundial. La difusión mediante sistemas mundiales como los medios, el derecho -especialmente en cuanto a los derechos humanos-, la actividad científica y la naciente actividad política de una sociedad civil global han generado posibilidades de posicionamiento que el movimiento mapuche está utilizando intensamente. 
Uno de los rendimientos de la conexión global de los posicionamientos mapuches es la "trans"-nacionalización del alcance discursivo y de las estructuras de movilización del movimiento. En efecto, el movimiento mapuche se mueve rápidamente hacia una reconstrucción simbólica y operativa de un espacio pan-mapuche, el Wallmapu como un todo del territorio ancestral mapuche en ambos lados de los Andes del Sur. De lado del discurso, la semántica del Wallmapu ha llegado a ser una unidad imaginaria de los diferentes discursos, estructuras sociales y redes de la identidad mapuche. La representación del Wallmapu pretende alcanzar todo lo que la identidad mapuche envuelve. Según Boccara (2006: 3),

construido sobre la investigación académica etnológica e histórica como en los testimonios y memorias de los mayores, involucra la existencia de redes indígenas que atravesaban la Araucanía, las Pampas y la Patagonia Norte. Esto enfatiza la existencia de una red mapuche transcontinental que usó múltiples alianzas matrimoniales, políticas y económicas para articular ambos lados de los Andes. De acuerdo a esta visión alternativa del Cono Sur, no hubo otros límites del territorio indígena que los océanos Atlántico y Pacifico.

Esta nueva representación discursiva Pan-mapuche, en la cual los Andes desaparecen como límite natural, desafía como geopolítica contrahegemónica a las representaciones territoriales de los Estados. Esto realiza un espacio de encuentro que significa una resemantización del territorio respecto a las semánticas religiosas y étnicas.

Posicionamientos sobre los derechos.

La protesta mapuche tiene, al igual que la protesta indígena global, como uno de sus componentes fundamentales el reclamo de derechos, no sólo del respeto cabal de los derechos individuales, sino también de derechos colectivos (Toledo 2006b). Dichos derechos corresponden a posiciones fundamentales de los sujetos en el orden jurídico que funcionan como mandatos de optimización (Alexy 2007). Como tales, esos derechos buscan asegurar institucionalmente mediante el sistema jurídico elementos básicos para la capacidad de autoconstituir su propia identidad por parte del pueblo mapuche. El alcance de esos derechos no refiere sólo al Estado, sino también frente a los privados, sobre todo empresas que amenazan los territorios y 
recursos naturales con los cuales los mapuches se han considerado especialmente vinculados.

La protesta mapuche sirve para hacer evidente la pobreza y vulnerabilidad de grupos marginalizados e indicar la falta de diversidad en sus órdenes semánticos, mientras que la exigencia de derechos busca hacer de nuevo procesables para los sistemas sociales esos efectos de exclusión mediante estructuras inclusivas en el sistema jurídico. La protesta puede ser estabilizada y renovada en el tiempo con nuevos temas (desde reclamos de tierra hasta protestas contra la biopiratería y la traducción unilateral de Windows MS en Mapudzungun) que incrementan la complejidad interna del movimiento. Los destinatarios de esa protesta son los intereses privados y los gobiernos que los apoyan y frente a ellos se reivindican derechos fundamentales colectivos que mejoren la posición de los mapuches en los diversos sistemas sociales. Las fuentes de movilización de este movimiento social son asunciones fuertes, tanto normativas como descriptivas. Por una parte, los discursos del movimiento mapuche convergen en demandas de derechos multiculturales (autodeterminación relativa, auto-administración, validez del derecho mapuche), derechos territoriales (acceso a los recursos naturales y espacios naturales y control compartido de estos) y derechos culturales (reconocimiento y apoyo para el propio lenguaje indígena, así como para las prácticas religiosas, médicas, educativas y otras prácticas étnicas). Esto implica modificaciones para adecuar las leyes a los derechos humanos de los pueblos indígenas y sus conceptos subyacentes de justicia multicultural.

Las organizaciones mapuches han protestado contra las compañías privadas y contra los gobiernos que han impulsado la forma de desarrollo guiada por los programas neoliberales de la política. En el caso de Chile, aquello ha tenido mayor intensidad.

Las políticas neoliberales en Chile implicaron la desregulación de mercados, incluyendo los recursos naturales que pudieron ser explotados libremente. Este es el caso de los recursos forestales que formaron parte de una economía basada en exportaciones de bajo valor agregado y en la explotación intensiva de recursos naturales. Desde 1975, los monocultivos pudieron expandirse en la zona mapuche. En años recientes, esta economía alcanzó una extensión de cerca de 1.500 .000 hectáreas en tierras ancestrales mapuches. La economía forestal se concentra en las manos de dos compañías, Mininco y Forestal Arauco, cuyos propietarios son los dos mayores grupos económicos chilenos. Para el 2010 se espera que alcance la extensión de 2,6 millones de hectáreas (OLCA 2002). Las proyecciones para 2020 llegan a 5 millones 
de hectáreas, cifra que podría aumentar debido al acuerdo de libre comercio que Chile firmó con China, que es el primer cliente de celulosa del país.

Los temas de protesta incluyen: La substitución del bosque nativo y el deterioro de la biodiversidad, desaparición de las plantas medicinales tradicionales, erosión de suelos debido a tala irrestricta y sus consecuencias para la agricultura y pastoreo, polución de suelos y aguas por uso de químicos, producción de residuos industriales, deterioro de las vías de acceso a las comunidades y decrecimiento de la disponibilidad de agua debido al consumo de las plantaciones forestales (Cárdenas y Atileo 2006). Aquellos problemas han tenido a menudo por consecuencia el desplazamiento forzado, el cual no fue compensado con mayores oportunidades laborales (Toledo 2006a, Haughney 2006).

Las organizaciones mapuches en el Gulumapu agregaron a su protesta otros temas de redistribución, como las consecuencias de la privatización de las aguas, la proliferación de los vertederos de basura, la minería y pesca privadas, el alcance de la pobreza, falta de tierra y oportunidades, así como reclamos simbólicos de no discriminación y reconocimiento de la diferencia cultural (Ray 2007). La respuesta de los gobiernos chilenos fue doble. Por una parte, implementó diversas medidas redistributivas inconsistentes, sin reconocimiento de derechos y con un enfoque campesinista. Por otra, el movimiento mapuche fue tratado con una dura represión y una amplia criminalización.

En Argentina, la crisis económica, social y política desde 2001 desplazaron los reclamos culturales a un lugar secundario respecto a la supervivencia social (Kropff 2005). Sin embargo, el nuevo contexto de movilizaciones múltiples y coordinadas de los movimientos sociales en los espacios públicos abrió nuevas posibilidades al activismo mapuche en el Puelmapu. Los avances en la coordinación de diferentes movimientos sociales promovieron la constitución de organizaciones nacionales como la ONPIA (Organización Nacional Argentina de Pueblos Indígenas), la cual ha alcanzado cierta participación en las decisiones políticas. Las organizaciones mapuches en el nuevo contexto son más hábiles para establecer alianzas con otros movimientos sociales. Además, ellas pueden interactuar directamente con organizaciones interestatales como el BID y el Banco Mundial. Entretanto, el movimiento mapuche debe confrontar diversos conflictos con actores privados que desean acceso a la tierra y a los recursos naturales de las comunidades. Los conflictos tales como la controversia judicial con Benetton han tenido resonancia nacional e inclusive mundial. Además de los reclamos de tierras (en 
localidades como Buenos Aires, Río Negro, Chubut, Neuquén, Cuyo), las comunidades mapuches confrontaron las pretensiones de las empresas petroleras norteamericanas Pioneer Natural Resources y Apache Corporation, así como la española Repsol en Neuquén, empresas mineras en Chubut, proyectos hidroeléctricos en Esquel, megaproyectos de turismo, etc. (Ray 2007).

Uno de los elementos destacables del posicionamiento de la protesta mapuche respecto de los derechos ha sido la mantención de los vínculos entre las demandas redistributivas y de reconocimiento. Muchas organizaciones del Gulumapu establecieron en los noventa el esquema interpretativo de la "deuda histórica" del Estado chileno respecto del pueblo mapuche. Este concepto relaciona los temas redistributivos y de reconocimiento en la protesta mapuche. Tales conceptos han penetrado inclusive en los reclamos de las organizaciones del Puelmapu. Este marco interpretativo posiciona al pueblo mapuche como sujetos moralmente intitulados respecto al sistema político en ambos lados del Wallmapu, y posiciona al establishment político como deudor de un trato justo con los mapuches.

\section{Posicionamiento sobre los medios de comunicación.}

Uno de los elementos más innovadores de la protesta mapuche ha sido su posicionamiento en cuanto a los medios de comunicación, debido a que han debido buscar desde el inicio medios alternativos eficaces para superar la exclusión de la comunicación política mapuche de la esfera pública oficial. La búsqueda de espacios y formas de comunicación alternativos ha sido uno de los rasgos de esta política de identidad. Este es todavía un terreno relativamente virgen a la investigación, sobre todo cómo los nuevos formatos y espacios de comunicación pueden tener efectos sobre los posicionamientos anteriores.

Queremos destacar una histórica e intensiva ciberpolitización de la política de la identidad mapuche. Para el movimiento mapuche del Wallma$p u$, los nuevos medios, especialmente Internet, han llegado a ser un sustrato privilegiado para la política étnica. El uso que han hecho los mapuches de Internet es sorprendente. Los activistas mapuches, de acuerdo a Salazar (s/f: 10), han adoptado esos medios "como un intento de reconstruir el discurso mapuche y el conocimiento en orden a mediar entre las discontinuidades en el tiempo y el espacio y para combatir el prejuicio. La red ha hecho posible la circulación de imágenes no disponibles en ningún otro lugar (como en el caso de los niños de Boyeco)". 
En un contexto de conflicto con los gobiernos y las empresas privadas, la red construye progresivamente una esfera pública alternativa que permite la circulación simultánea de noticias, manifiestos, papers, imágenes y video, cosa que no permitían los medios tradicionales. Esta esfera pública alternativa ofrece nuevas vías de narrar y construir un nuevo imaginario mapuche (Salazar 2003). La red favorece el florecimiento de múltiples formas de representación para la identidad mapuche: Vías tradicionales, esencialistas y reflexivas compiten y pueden ser mezcladas en la red (Godoy 2003).

Por otro lado, se puede apreciar una especialización de nuevas formas de producción cultural de la diferencia mapuche. La emergencia de un movimiento étnico mapuche fue acompañada con la visible irrupción de una poesía mapuche en el panorama de la literatura (Park 2007), la que puede ser vista como una respuesta a la hegemonía de discursos occidentales en la literatura nacional (García 2006). Sin embargo, esta poesía constituye un discurso intercultural, una hibridación, generada en interacción con los discursos de la literatura chilena (Carrasco 2006) y muestra procedimientos transtextuales e interculturales: involucra el canon europeo occidental usado como un soporte para sus propios elementos culturales (epeu, ül y konew), es decir, su propio sistema textual, en el cual se emplaza un nuevo discurso indígena que dialoga críticamente con el contexto chileno (García 2008). La nueva poesía mapuche, a menudo bilingüe, tiene un carácter expresivo, testimonial y performativo. Esto ha desarrollado un hato de estrategias textuales (enunciación sincrética, intertexto transliterario y codificación plural), la aparición de nuevos géneros literarios en Mapudzungun, tales como historias personales, el epeu didáctico, el ensayo etnográfico y el poema escrito (Carrasco 2000).

Adicionalmente, se pueden observar formas emergentes de expresión musical mapuche. La multiplicación de grupos musicales en el contexto urbano que se dedican al Rock, la música electrónica, la música fusión mapuche de todo tipo (especialmente Reggae, Hip-Hop) y su difusión a través de canales alternativos, crean un incipiente discurso híbrido que dialoga con muchos géneros musicales, usa temas y elementos propios (ritmos e instrumentos) y traspasa los límites del folklore mapuche tradicional (que introduce instrumentos y formas occidentales como la orquestación) y de la canción tradicional mapuche (canciones ceremoniales de la machi, canciones protocolares y de saludo, canciones improvisadas en ocasiones sociales). Un exponente de esta fusión dijo: 
Cuando hablamos de 'Mapuchizar' el hip-hop y la poesia, nosotros queremos decir incorporarlo en nuestra cultura. A través de esas formas artísticas, nosotros llevamos a la luz nuestras luchas personales y colectivas. Podriamos hablar de una etnopoesia y un etno hiphop, conectados en este caso con el pueblo mapuche (Estrada 2006).

El uso de herramientas audiovisuales es una forma muy incipiente de producción cultural. Desde la producción de Wiñometun nimapu meu de José Ancán y Hernán Dinamarca en 1994, se observa un documentalismo mapuche. Artistas femeninas como Sofía Painequeo y Jeannette Paillán han realizado sus propias producciones, donde dialogan con los estereotipos visuales que la producción documental chilena había establecido previamente en relación a la forma de vida mapuche (Paz 2008).

\section{Conclusión}

Los posicionamientos descritos nos permiten ver cómo la teoría de sistemas puede servir de marco teórico a investigaciones culturales y de qué manera la política de la identidad que caracteriza a la evolución reciente de las formas culturales no es sólo real respecto a sus posibles efectos de inclusión sino también para la producción de la comunicación de los sistemas sociales. La comunicación de las organizaciones mapuches como sistema social de protesta consta de una serie de posicionamientos que estructuran formas de comunicación conectadas con los sistemas funcionales, especialmente con el sistema político que ha tenido que referirse muchas veces a la realidad que la comunicación de protesta indígena en el Cono Sur está generando. En este perspectiva, contexto social e institucional -la diferenciación social con primado funcional- y comunicación cultural aparecen como recíprocamente condicionados aunque analítica y estructuralmente diferenciados. Cómo se relaciona la comunicación de protesta con las formas de identificación cotidianas de los mapuches, o cómo los sistemas funcionales afectan dichas comunicaciones cotidianas, no ha sido analizado aquí y debe ser objeto de futuras investigaciones, así como también un estudio de la interrelación entre protesta indígena y sistemas funcionales. Tampoco se ha buscado hacer un análisis sistemático de la realidad mapuche a la luz de la teoría de sistemas sino apenas hacer una ilustración de cuáles podrían ser los puntos de partida de un análisis sistémico de ella.

Como podemos ver, los discursos y estructuras de la política de la identidad en el movimiento mapuche están cambiando los bordes, diagramas y 
jerarquías de la identidad mapuche, así como la relación con las identidades nacionales en Argentina y Chile. Los cinco tipos de posicionamiento de la protesta mapuche que han sido expuestos narran fenómenos conocidos de la comunicación mapuche, pero conectándolos con conceptos de discursos, semántica y estructura de la teoría de los sistemas sociales, permitiéndonos ilustrar cómo dicha teoría puede empezar a ser usada fructíferamente para el análisis cultural. En especial, permite el análisis sistémico reparar cómo las formas de diferenciación cultural se relacionan con estructuras que derivan de la diferenciación funcional de la sociedad, de manera que la contingencia de ambas evoluciona en una covariación, tal y como lo muestran cada uno de los posicionamientos.

En ese sentido, el movimiento mapuche como referencia identitaria es afectado por la contingencia y productor de ella. Ello también envuelve la complejidad de los nuevos medios y las formas de producción cultural y la generación de nuevos espacios étnicos en dicha esquina de la sociedad mundial. La disolución de las formas tradicionales de vida que determina la diferenciación funcional, conforma el cimiento de la evolución de las etnicidades y la transformación de las representaciones identitarias y las comunicaciones políticas relacionadas. La etnicidad está lejos de perder importancia en la sociedad mundial, pero juega actualmente un rol central en el incremento de la complejidad de la comunicación. El esquema comparativo de las identidades culturales no puede ser considerado vacío en dichos procesos. Por el contrario, ellas expanden y multiplican las formas de vida diferenciadas, las cuales son parte de la sociedad moderna.

La función de la política de la identidad mapuche como movimiento de protesta que critica los vacíos y fallas de la evolución del sistema funcional de la política en el Cono Sur, y sus acoplamientos problemáticos con otros sistemas sociales como el derecho, la economía y los medios, hacen las demandas mapuches no sólo analíticamente ricas, sino indispensables para el análisis en términos de la teoría de sistemas del desarrollo poscolonial en sistemas sociales en la región sur de la sociedad mundial. 


\section{BiBLIOGRAFÍA}

Alcoff, L. 2000. "Who's Afraid of Identity Politics?". En Reclaiming Identity, Realist Theory and the Predicament of Postmodernism, editado por M. L. Moya y Michael R. Hames-García, pp: 312-313. University of California Press, Berkeley.

Alexy, R. 2007. Teoría de los Derechos Fundamentales. 2a edición. Centro de Estudios Constitucionales, Madrid.

Antileo, E. 2006. Mapuches santiaguinos: posiciones y discusiones del movimiento mapuche en torno al dilema de la urbanidad. Manuscrito disponible en http://meli.mapuches.org/img/pdf/mapuche_urbanos_ para.pdf

Aravena, A. 1999. "Identidad indígena en los medios urbanos: procesos de recomposición de la identidad étnica mapuche en la ciudad de Santiago". En Lógica mestiza en América, editado por Guillaume Boccara y Silvia Galindo, pp: 165-199. Instituto de Estudios Indígenas, Universidad de la Frontera, Temuco.

Baecker, D. 2003. Wozu Kultur? Kulturverlag Kadmos, Auflage.

Baecker, D. 2008. "Zur Kontingenzkultur der Weltgesellschaft". En Über Kultur. Theorie und Praxis der Kulturreflexion, editado por Dirk Baecker, Matthias Kettner y Dirk Rustemeyer, pp: 139-161. Transcript, Bielefeld.

Bello, A. 2002. "Migración, identidad y comunidad mapuche en Chile: entre utopismos y realidades". Asuntos Indígenas 3-4: 40-47.

Bengoa, J. 1991. Historia del Pueblo Mapuche en los siglos XIX y XX. 2a edición. Lom, Santiago de Chile.

Benhabib, S. 2002. The claims of culture, equality and diversity in the global era. Princeton University Press, Princeton/Oxford.

Bhabha, H. 1990. "DissemiNation: Time, Narrative, and the Margins of the Modern Nation". En Nation and Narration, editado por Homi Bhabha, pp: 291-322. Routledge, London/New York.

Boccara, G. 2006. "The brighter side of the indigenous renaissance (Part 2)". Nuevo Mundo Mundos Nuevos, Debates. Disponible en http:// nuevomundo.revues.org/index $2483 . \mathrm{html}$. 
Bora, A. 1999. Differenzierung und Inklusion, partizipative Öffentlichkeit im Rechtssystem moderner Gesellschaften. Nomos, Baden-Baden.

Bora, A. 2008. "System, Diskurs, Position, Überlegungen zur Theorie sozialer Differenzierung". Ponencia presentada en el post-graduate workshop Semantik als Grundbegriff der Soziologie? Junio 2008. Bielefeld.

Bretho, M. y R. Iriarte. 2003. "Ser mapuche en un contexto posreduccional. Las mil caras de la etnicidad". Cyber Humanitatis 27. Disponible en http://www.cyberhumanitatis.uchile.cl/CDA/texto_si mple2/0,1255,SCID\%253D7521\%2526ISID\%253D347,00.html

Briones, C. 2006. "Cuestionando geografías estatales de inclusión en Argentina. La política cultural de organizaciones con filosofía y liderazgo Mapuche". En Cultural Agency in the Americas: Language, Ethnicity, Gender and Outlets of Expression, editado por Doris Sommer, pp: 248-280. Duke University Press, 2006

Briones, C. 2007. "Teorías performativas de la identidad y performatividad de las teorías". Taula Rasa 6: 55-83.

Butler, J. 1990. Gender Trouble: Feminism and the Subversion of Identity. Routledge, New York.

Cárdenas, A. y E. Antileo. 2006. "Plantaciones forestales en Chile y su relación con el pueblo mapuche". Ponencia Presentada en el Foro Social de Resistencia contra los Agronegocios. Junio 2006. Buenos Aires.

Carrasco, I. 2000. "Poetas mapuches en la literatura chilena". Estudios Filológicos 35: 139-149.

Carrasco, H. 2006. "Poesía mapuche actual: la identidad escindida. Viaje al osario de Juan Paulo Huirimilla". Revista Chilena de Literatura 68: 141-168.

Davies, B. y R. Harre. 1990. "Positioning: The Discursive Production of Selves". Journal for Theory and Social Behaviour 20 (1): 43-63.

Díaz-Polanco, H. 1997. Indigenous Peoples in Latin America: The Quest for Self-Determination. Westview, Boulder.

Escobar, A. 1992. "Culture, practice and politics: anthropology and the study of social movements". Critique of Anthropology 12(4): 395-432. 
Espósito, E. 2002. Soziales Vergessen: Formen und Medien des Gedächntnisses der Gesselschaft. Suhrkamp, Frankfurt am Main.

Estrada, D. 2006. "Mapuche Hip Hop". Internet Press Service, 24 de octubre. Disponible en http://ipsnews.net/news.asp?idnews=35211

Foerster, R. 1999. "Movimiento étnico o movimiento etno-nacional". Revista de Critica Cultural 18: 52-58.

Foerster, R. 2001. "Sociedad Mapuche y Sociedad Chilena: La Deuda Histórica”. Polis, Revista de la Universidad Bolivariana 1(2). Disponible en http://www.revistapolis.cl/polis\%20final/2/foert.htm

Foerster, R. y S. Montecino 1988. Organizaciones, Lideres y Contiendas mapuches 1910-1970. Centro de Estudios de la Mujer, Santiago de Chile.

García, M. 2006. "El discurso poético mapuche y su vinculación con los "temas de resistencia cultural". Revista Chilena de Literatura 68: 169197.

García, M. 2008. "Entre-textos: la dimensión dialógica e intercultural del discurso poético mapuche". Revista Chilena de Literatura 72: 29 - 70.

Godoy, C. 2003. "Sitios mapuches en Internet: Reimaginando la identidad". Revista Chilena de Antropología Visual 3: 59-83.

Haughney, D. 2006. Neoliberal Economics, Democratic Transition, and Mapuche Demands for Rigths in Chile. The University Press of Florida, Gainesville

Hellman, K. 1996. Systemtheorie und neue soziale Bewegungen, Identitätsprobleme in der Risikogesellschaft. Westdeutscher Verlag, Opladen

Kotov, R. y J. Vergara. 1995. "La identidad mapuche en la perspectiva de los intelectuales indígenas". Actas del Segundo Congreso Chileno de Antropologia, Tomo I, pp: 453-461. Valdivia.

Kropff, L. 2005. "Activismo mapuche en Argentina: trayectoria histórica y nuevas propuestas". En Pueblos indígenas, Estado y democracia, compilado por P. Dávalos, pp: 103-132. Clacso, Buenos Aires. 
Le Bonniec, F. 2002. "Las Identidades Territoriales o Cómo Hacer Historia desde hoy día". En Territorialidad Mapuche en el siglo XX, compilado por R. Morales, pp: 31-49. Ediciones Escaparate, Temuco.

Leon, L. 2007. “Ngulan Mapu (Araucanía): La 'pacificación' y su relato historiográfico, 1900-1973". Revista de Historia Social y de las Mentalidades XI (2): 137-170.

Luhmann, N. 1980. "Gesellschaftliche Struktur und semantische Tradition". En Gesellschaftsstruktur und Semantik, Studien zur Wissenssoziologie der modernen Gesellschaft, Band 1, compilado por N. Luhmann, pp: 9-72. Suhrkamp, Frankfurt am Main.

Luhmann, N. 1984. Soziale Systeme. Suhrkamp, Frankfurt am Main.

Luhmann, N. 1995. Die Kunst der Gesellschaft. Suhrkamp, Frankfurt am Main.

Luhmann, N. 1997. Die Gesellschaft der Gesellschaft. Suhrkamp, Frankfurt am Main.

Luhmann, N. 2008. Ideenevolution. Suhrkamp, Frankfurt am Main.

Marimán, J. 2003. "Identidad Fragmentada". Periódico Mapuche Azkintuwe 2. Diciembre. Disponible en http://www.mapuche-nation. org/espanol/html/articulos/art-41.htm

Mascareńo, A. 2007a. "Sociología de la Cultura, la Deconstrucción de lo Mapuche”. Estudios Públicos 105: 61-112.

Mascareño, A. 2007b. "La cultura chilena como ficción real". En El Chile del Bicentenario: aportes para el debate, editado por Manuel Vicuña, pp: 183-220. Universidad Diego Portales, Santiago.

Millán, M. 2008. "La Construcción de la Identidad mapuche en contextos urbanos y rurales en el Wallmapu, Argentina". En Identidades, Etnocidio y Racismo en América Latina, editado por F. García, pp: 159-179. Flacso, Quito.

Moghaddam, F. 2007. Multiculturalism and Intergroup Relations: Psychological Implications for Democracy in Global Context. American Psychological Association, Washington, D.C. 
Nassehi, A. 1990. "Zum Funktionswandel von Ethnizität im Prozess gesellschaftlicher Modernisierung, ein Beitrag zur Theorie funktionaler Differenzierung". Soziale Welt 41(3): 261 - 282.

Nassehi, A. 2006. "Dialog der Kulturen - wer spricht?" Aus Politik und Zeitgeschichte 28-29: pp. 33-38.

OLCA (Observatorio Latinoamericano de Conflictos Ambientales). 2002. Plantaciones forestales y comunidades mapuches. OLCA, Santiago.

Park, J. 2007. "Discursos y poética mapuche-huilliche actual: Cambio generacional y diferencia territorial". Alpha 24: 139-162.

Paz, M. 2008. "La Cámara en manos del otro. El estereotipo en el video indígena mapuche". Revista Chilena de Antropología Visual 12: 71-102.

Peyser, A. 2003. Desarrollo, Cultura e Identidad. "El caso del mapuche urbano en Chile». Elementos y estrategias identitarias en el discurso indigena urbano. Tesis para optar al grado de Doctor en Cs. Sociales, Université Catholique de Lovaine, Lovaine.

Ray. L. 2007. The Language of the Land, Mapuche in Argentina and Chile. International Work Group for Indigenal Affairs IWGIA, Document 119 , Copenhagen.

Quijano, A. 2000. "Coloniality of Power, Eurocentrism, and Latin America”. Nepantla: Views from South 1 (3): 533-580.

Salazar, G. 1999. Historia Contemporánea de Chile, Vol. II. Lom, Santiago.

Salazar, J. 2003. "Articulating an activist imaginary: Internet as counter public sphere in the Mapuche movement". Media Information Australia incorporating Culture and Policy 107: 19-29.

Salazar, J. (s/f). Digital media as collaborative research: the case of Mapuche media in Chile. Manuscrito disponible en: http://lists.ou.edu/cgibin/wa?A3=ind $0405 \& \mathrm{~L}=$ OMIVPROGRAM $\& \mathrm{E}=$ base $64 \& \mathrm{P}=492$ $168 \& \mathrm{~B}=-$-Apple-Mail-10--63555195\&T=application $\% 2 \mathrm{Fpdf} ; \% 20$ name $=\% 22$ salazarIAMCR.pdf\%22\&N=salazarIAMCR.pdf

Schroer, M. 2010. "Kultursoziologie". En Handbuch Spezielle Soziologien, editado por G. Kneer y M. Schroer, pp: 197-220. Verlag für Sozialwissenschaften, Wiesbaden. 
Stäheli, U. 2000. Sinnzusammenbrüche, eine dekonstruktive Lektüre von Niklas Luhmanns Systemtheorie. Velbrück Wissenschaft, Göttingen.

Stichweh, R. 2000. "Semantik und Sozialstruktur: Zur Logik einer systemtheoretischen Unterscheidung”. Soziale Systeme 6: 237-250.

Toledo, V. 2006a. Pueblo mapuche, derechos colectivos y territorio: Desafíos para la sustentabilidad democrática. Lom, Santiago de Chile.

Toledo, V. 2006b. "La memoria de las tierras antiguas tocando a las puertas del derecho. Políticas de la memoria mapuche en la transición chilena". En El Derecho a la Memoria, compilado por F. Gómez, pp: 421-440. Universidad de Deusto, Diputación Foral de Gipuzkoa, Bilbao.

Thompson, J. 2002. Ideología y cultura modernas. 2a. Edición. UAM, México.

Urry, J. 2002. Mobilities and Connections. Manuscrito disponible en http:// www.ville-en-mouvement.com/telechargement/040602/mobility.pdf

Valdés, M. 2007. Pueblos Originarios y Movimientos Sociales. Manuscrito disponible en http://www.mapunet.org/documentos/mapuches/ movimientos_sociales_pueblos_originarios.pdf

Van Bebber, R. 2002. "Estado-Nación y "conflicto mapuche": aproximación al discurso de los partidos políticos chilenos". En Crisis y Conflicto en el Capitalismo Latinoamericano, editado por L. Levy, pp: 325-355. Clacso, Buenos Aires.

Wade, P. 1997. Race and Ethnicity in Latin America. Pluto Press, London.

Yashar, D. 2005. Contesting Citizenship in Latin America. The Rise of Indigenious Movements and the Postliberal Challenge. Cambridge University Press, Cambridge.

Young, I. 1997. "Difference as Ressource for Democratic Communications". En Deliberative Democracy, Essays on Reason and Politics, editado por J. Bohman y W. Rehg, pp: 383-406. The MIT Press, Boston.

Young, I. 2000. Inclusion and Democracy. Oxford University Press, Oxford.

Zimmerman, C. 2003. "Die Kultur der Systemtheorie". En Theorie und Metatheorie, Reflexionen aufpolitikwissenschaftliche Motive, editado por J. Klawitter, M. Gsänger y C. Zimmerman, pp: 43-69. Königshausen und Neumann, Würzsburg. 